\title{
New Soliton-like Solutions of Variable Coefficient Nonlinear Schrödinger Equations
}

\author{
Heng-Nong Xuan, Changji Wang ${ }^{\mathrm{a}}$, and Dafang Zhang ${ }^{\mathrm{b}}$ \\ College of Information Engineering, Nanjing University of Finance and Economics, \\ Nanjing 210003, China \\ a Network Research Center, Tsinghua University, Beijing 100084, China \\ ${ }^{\mathrm{b}}$ School of Computer and Communication Technology, Hunan University, Changsha 410082, China \\ Reprint requests to H.-N. X.; E-mail: hn_xuan@yahoo.com.cn
}

Z. Naturforsch. 59a, 196-202 (2004); received January 20, 2004

The improved projective Riccati system method for solving nonlinear evolution equations (NEEs) is established. With the help of symbolic computation, one can obtain more exact solutions of some NEEs. To illustrate the method, we take the variable coefficient nonlinear Schrödinger equation as an example, and obtain four families of soliton-like solutions. Eight figures are given to illustrate some features of these solutions.

Key words: Variable Coefficient Schrödinger Equation; Soliton-like Solutions; Symbolic Computation.

\section{Introduction}

Nonlinear evolution equations (NEEs) are applied in physics as well as chemistry and biology. It is important to obtain exact solutions of these equations. Many powerful methods have been presented, such as the Backlund and the Darboux transformation [1], the sine-cosine method [2,3], various tanh methods [4-7], the generalized Riccati equation expansion [8], and the hyperbolic function method [9]. Recently some methods based on symbolic computation have been developed.

In this paper we improve the projective Riccati system method, which is established for constructing soliton-like multiple travelling wave solutions for some NEEs [10-14] and we choose the variable coefficient Schrödinger equation [15] to illustrate the improved method.

In Sect. 2 we propose the improved projective Riccati equations method and in Sect. 3 we apply the method to the variable coefficient nonlinear Schrödinger (VCNLS) equation and find four families of exact soliton-like solutions. Finally a short summary is presented.

\section{The Improved Projective Riccati System Method}

We establish the improved projective Riccati system method, given a NEE with, say, two variables $x$ and $t$,

$$
E\left(u_{t}, u_{x}, u_{x t}, u_{t t}, u_{x x}, \cdots\right)=0,
$$

in three steps.

Step 1. We express the solutions of (1) as

$$
u(x, t)=a_{0}+\sum_{i=1}^{m} \sigma^{i-1}(\xi)\left[a_{i} \sigma(\xi)+b_{i} \tau(\xi)\right],
$$

where $a_{0}=a_{0}(x, t), a_{i}=a_{i}(x, t), b_{i}=b_{i}(x, t), \quad(i=$ $1, \cdots, m)$, and $\xi=\xi(x, t)$ are all unknown functions of $x$ and $t$, and $\sigma(\xi)$ and $\tau(\xi)$ satisfy the following projective Riccati system [10 - 14]

$$
\begin{aligned}
\sigma^{\prime}(\xi) & =\varepsilon \sigma(\xi) \tau(\xi), \tau^{\prime}(\xi)=R+\varepsilon \tau^{2}(\xi)-\mu \sigma(\xi) \\
\varepsilon & = \pm 1
\end{aligned}
$$

$\tau^{2}(\xi)=-\varepsilon\left[R-2 \mu \sigma(\xi)+\frac{\mu^{2}-1}{R} \sigma^{2}(\xi)\right], R \neq 0$,

where $R, \mu$ are constants and ${ }^{\prime}=\mathrm{d} / \mathrm{d} \xi$.

The parameter $m$ is the balance constant, which is obtained by balancing the highest order derivative term and the nonlinear terms in (1) ( $m$ is usually a positive integer). If $m$ is a fraction or a negative integer, we usually make the transformation $u(x, t)=v^{m}(x, t)$ firstly.

It is necessary to point out that in $[10,12-14]$ the coefficients $a_{0}, a_{i}, b_{i}$ are all constants and $\xi$ is a linear function of $x$ and $t$.

Step 2. Substituting (2) along with (3) and (4) into (1) we can obtain a set of algebraic polynomials 
for $\tau^{i}(\xi) \sigma^{j}(\xi)(i=0,1 ; j=0,1, \cdots)$ from the numerator of the resulting system. Setting the coefficients of these terms $\tau^{i}(\xi) \sigma^{j}(\xi)$ to zero, we get a system of over-determined partial differential equations (PDEs) or ordinary differential equations (ODEs) with respect to unknown functions of $a_{0}, a_{i}, b_{i}(i=1, \cdots, m)$ and $\xi$.

Step 3. Solving the above system by use of the symbolic computation system-Maple, we would end up with the explicit expressions for $\mu, a_{0}, a_{i}, b_{i}$ ( $i=$ $1, \cdots, m)$ and $\xi$ or the constraints among them.

In order to solve the PDEs (ODEs) derived in Step 2 easily, we may choose special forms of $a_{i}, b_{i}$ and $\xi$ on a trial-and-error basis.

We know that the projective Riccati system (3) - (4) has the following solutions:

Case 1. When $\varepsilon=-1$ :

$\sigma_{1}(\xi)=\frac{R \operatorname{sech}(\sqrt{R} \xi)}{\mu \operatorname{sech}(\sqrt{R} \xi)+1}, \tau_{1}(\xi)=\frac{\sqrt{R} \tanh (\sqrt{R} \xi)}{\mu \operatorname{sech}(\sqrt{R} \xi)+1}$,

$\sigma_{2}(\xi)=\frac{R \operatorname{csch}(\sqrt{R} \xi)}{\mu \operatorname{csch}(\sqrt{R} \xi)+1}, \tau_{2}(\xi)=\frac{\sqrt{R} \operatorname{coth}(\sqrt{R} \xi)}{\mu \operatorname{csch}(\sqrt{R} \xi)+1}$.

Case 2. When $\varepsilon=1$ :

$$
\begin{gathered}
\sigma_{3}(\xi)=\frac{R \sec (\sqrt{R} \xi)}{\mu \sec (\sqrt{R} \xi)+1}, \tau_{3}(\xi)=\frac{\sqrt{R} \tan (\sqrt{R} \xi)}{\mu \sec (\sqrt{R} \xi)+1}, \\
\sigma_{4}(\xi)=\frac{R \csc (\sqrt{R} \xi)}{\mu \csc (\sqrt{R} \xi)+1}, \tau_{4}(\xi)=-\frac{\sqrt{R} \cot (\sqrt{R} \xi)}{\mu \csc (\sqrt{R} \xi)+1} .
\end{gathered}
$$

Thus according to (2), (5), (6) and the conclusions in Step 3, we can obtain many families of exact solutions for Eq. (1).

\section{Remarks}

1. The transformation (2)-(4) is more general than the transformations in the tanh method [4], various extended tanh-function methods [6,7], generalized hyperbolic-function method [9], generalized Riccati equation expansion method [8] and projective Riccati equations method [10-14]. Firstly, compared to the tanh method, various extended tanh-function methods as well as the projective Riccati equations method, the restriction on $\xi(x, t)$ as merely a linear function $\{x, t\}$ and the restriction on the coefficients $a_{0}, a_{i}$, $b_{i}(i=1, \cdots, m)$ as constants are removed. Secondly, when $\mu=0$ in (2)-(4), the generalized Riccati equation expansion method and the generalized hyperbolicfunction method can be recovered.

2 . We call the solutions (2) solitary wave solutions if they contain a variable $\xi=\xi(x, y)$ that linear is $x, t$, and if $a_{0}=a_{0}(x, t), a_{i}=a_{i}(x, t), b_{i}=b_{i}(x, t),(i=1, \cdots, m)$ are all constants. Otherwise we call them soliton-like solutions. (see $[8,9]$ for details).

\section{Exact Soliton-like Solutions}

The VCNLS equation can be expressed as

$$
i \psi_{t}+A(t) \psi_{x x}+B(t)|\psi|^{2} \psi=0
$$

where $\psi=\psi(x, t)$ is a real or complex valued arbitrary function of $\{x, t\}$, and $A(t)$ and $B(t)$ are real valued arbitrary functions of $t$. [15] Khater et al. studied the conservation laws of (7) by Noether's theorem.

In order to obtain exact solutions of (7), firstly we make the transformation

$$
\psi(x, t)=u(x, t) \exp [i \theta(x, t)] .
$$

Then substituting (8) into (7) and setting the real and imaginary parts of the resulting equation equal to zero, we obtain the following sets of partial differential equations:

$$
\begin{aligned}
& -u \theta_{t}+A(t)\left(u_{x x}-u \theta_{t}^{2}\right)+B(t) u^{3}=0, \\
& u_{t}+2 A(t) u_{x} \theta_{x}+A(t) u \theta_{x x}=0 .
\end{aligned}
$$

Now we use the improved projective Riccati system method to seek some exact solutions of (9)-(10).

By balancing $u_{x x}$ and $u^{3}$ in (10), we obtain $m=1$ in (2). Therefore we assume the solutions of (9)-(10) in the following special forms:

$$
\begin{aligned}
& u(x, t)=a_{0}(t)+a_{1}(t) \sigma(\xi)+b_{1}(t) \tau(\xi), \\
& \xi=x p(t)+q(t), \\
& \theta(x, t)=x^{2} \lambda_{2}(t)+x \lambda_{1}(t)+\lambda_{0}(t),
\end{aligned}
$$

where $a_{0}(t), a_{1}(t), b_{1}(t), p(t), q(t), \lambda_{2}(t), \lambda_{1}(t)$ and $\lambda_{0}(t)$ are functions of $t$ to be determined, $\tau(\xi)$ and $\sigma(\xi)$ satisfying (3) $-(4)$.

Substituting (11) and (12) along with (3) and (4) into (9)-(10), collecting coefficients of monomials of $\tau(\xi), \sigma(\xi)$ and $x$ of the resulting system's numerator (Note that $A(t), B(t), a_{0}(t), a_{1}(t), b_{1}(t), p(t)$, 
$q(t), \lambda_{0}(t), \lambda_{1}(t), \lambda_{2}(t)$ are independent of $\left.x\right)$, then setting each coefficient to zero, under $\varepsilon=-1$ we obtain the following over-determined ODEs system with respect to differentiable functions $A(t), B(t), a_{0}(t), a_{1}(t)$, $b_{1}(t), p(t), q(t), \lambda_{0}(t), \lambda_{1}(t), \lambda_{2}(t):$

$$
\begin{aligned}
& -b_{1}(t)(\mu-1)(\mu+1) \\
& \cdot\left(\frac{\mathrm{d}}{\mathrm{d} t} q(t)+2 A(t) p(t) \lambda_{1}(t)\right)=0, \\
& -b_{1}(t)(\mu-1)(\mu+1) \\
& \cdot\left(4 \lambda_{2}(t) p(t) A(t)+\frac{\mathrm{d}}{\mathrm{d} t} p(t)\right)=0, \\
& -a_{1}(t) R\left(\frac{\mathrm{d}}{\mathrm{d} t} \lambda_{2}(t)+4\left(\lambda_{2}(t)\right)^{2} A(t)\right)=0, \\
& R\left[R A(t)(p(t))^{2} a_{1}(t)+3 R B(t) a_{1}(t)\left(b_{1}(t)\right)^{2}\right. \\
& -A(t) a_{1}(t)\left(\lambda_{1}(t)\right)^{2}+3 B(t)\left(a_{0}(t)\right)^{2} a_{1}(t) \\
& \left.\left(\frac{\mathrm{d}}{\mathrm{d} t} \lambda_{0}(t)\right) a_{1}(t)-6 B(t) a_{0}(t)\left(b_{1}(t)\right)^{2} \mu\right]=0, \\
& -a_{1}(t) R\left(4 A(t) \lambda_{2}(t) \lambda_{1}(t)+\frac{\mathrm{d}}{\mathrm{d} t} \lambda_{1}(t)\right)=0, \\
& -a_{0}(t) R\left(\frac{\mathrm{d}}{\mathrm{d} t} \lambda_{2}(t)+4\left(\lambda_{2}(t)\right)^{2} A(t)\right)=0, \\
& R a_{0}(t)\left(B(t)\left(a_{0}(t)\right)^{2}-\frac{\mathrm{d}}{\mathrm{d} t} \lambda_{0}(t)\right. \\
& \left.-A(t)\left(\lambda_{1}(t)\right)^{2}+3 R B(t)\left(b_{1}(t)\right)^{2}\right)=0, \\
& -a_{0}(t) R\left(4 A(t) \lambda_{2}(t) \lambda_{1}(t)+\frac{\mathrm{d}}{\mathrm{d} t} \lambda_{1}(t)\right)=0, \\
& -a_{1}(t) R\left(4 \lambda_{2}(t) p(t) A(t)+\frac{\mathrm{d}}{\mathrm{d} t} p(t)\right)=0, \\
& -a_{1}(t) R\left(\frac{\mathrm{d}}{\mathrm{d} t} q(t)+2 A(t) p(t) \lambda_{1}(t)\right)=0, \\
& \left(\frac{\mathrm{d}}{\mathrm{d} t} a_{0}(t)\right) R=0, \\
& R b_{1}(t)\left(R B(t)\left(b_{1}(t)\right)^{2}-A(t)\left(\lambda_{1}(t)\right)^{2}\right. \\
& \left.+3 B(t)\left(a_{0}(t)\right)^{2}-\frac{\mathrm{d}}{\mathrm{d} t} \lambda_{0}(t)\right)=0, \\
& -b_{1}(t) R\left(\frac{\mathrm{d}}{\mathrm{d} t} \lambda_{2}(t)+4\left(\lambda_{2}(t)\right)^{2} A(t)\right)=0, \\
& b_{1}(t) \mu R\left(4 \lambda_{2}(t) p(t) A(t)+\frac{\mathrm{d}}{\mathrm{d} t} p(t)\right)=0,
\end{aligned}
$$

$$
\begin{gathered}
b_{1}(t)\left(\left(b_{1}(t)\right)^{2} B(t) \mu^{2}-B(t)\left(b_{1}(t)\right)^{2}\right. \\
-2 A(t)(p(t))^{2}+3 B(t)\left(a_{1}(t)\right)^{2} R \\
\left.+2 \mu^{2}(p(t))^{2} A(t)\right)=0 \\
-6 B(t) a_{1}(t)\left(b_{1}(t)\right)^{2} \mu R+3 B(t) a_{0}(t)\left(a_{1}(t)\right)^{2} R \\
-3 A(t)(p(t))^{2} a_{1}(t) \mu R-3 B(t) a_{0}(t)\left(b_{1}(t)\right)^{2} \\
+3 B(t) a_{0}(t)\left(b_{1}(t)\right)^{2} \mu^{2}=0, \\
b_{1}(t) R\left(-2 B(t)\left(b_{1}(t)\right)^{2} \mu-\mu(p(t))^{2} A(t)\right. \\
\left.+6 B(t) a_{0}(t) a_{1}(t)\right)=0, \\
R \frac{\mathrm{d}}{\mathrm{d} t} b_{1}(t)=0, \\
-b_{1}(t) R\left(4 A(t) \lambda_{2}(t) \lambda_{1}(t)+\frac{\mathrm{d}}{\mathrm{d} t} \lambda_{1}(t)\right)=0, \\
R\left[2 \mu b_{1}(t) \lambda_{1}(t) p(t) A(t)\right. \\
\left.+\left(\frac{\mathrm{d}}{\mathrm{d} t} q(t)\right) \mu b_{1}(t)+\frac{\mathrm{d}}{\mathrm{d} t} a_{1}(t)\right]=0, \\
a_{1}(t)\left(3\left(b_{1}(t)\right)^{2} B(t) \mu^{2}+B(t)\left(a_{1}(t)\right)^{2} R\right. \\
+2 \mu^{2}(p(t))^{2} A(t)-2 A(t)(p(t))^{2} \\
\left.-3 B(t)\left(b_{1}(t)\right)^{2}\right)=0 .
\end{gathered}
$$

Solving (13) - (33) by Maple, we obtained the following results:

Case 1. In this case, $B(t)$ is considered to be an arbitrary function of $t$ with $\mu=a_{1}(t)$

$$
\begin{gathered}
\mu=a_{1}(t)=a_{0}(t)=\lambda_{2}(t)=0, \\
\lambda_{1}(t)=C_{2}, \quad A(t)=-\frac{1}{2} \frac{C_{1}^{2} B(t)}{C_{3}^{2}}, \\
\lambda_{0}(t)=\frac{1}{2}\left[2 C_{1}^{2} \int B(t) \mathrm{d} t C_{3}^{2} R+C_{1}^{2} \int B(t) \mathrm{d} t C_{2}^{2}\right. \\
\left.\quad+2 C_{4} C_{3}^{2}\right] / C_{3}^{2}, \\
q(t)=\frac{C_{1}^{2} C_{2} \int B(t) \mathrm{d} t+C_{5} C_{3}}{C_{3}}, \\
b_{1}(t)=C_{1}, \quad p(t)=C_{3},
\end{gathered}
$$

where $R$ and $C_{1}, \cdots, C_{5}$ are all arbitrary constants. 
Case 2. In this case, $B(t)$ is considered to be an arbitrary function of $t$ with $\mu=b_{1}(t)$

$$
\begin{gathered}
\mu=b_{1}(t)=a_{0}(t)=\lambda_{2}(t)=0, \\
q(t)=-\frac{C_{1}^{2} R C_{2} \int B(t) \mathrm{d} t-C_{5} C_{3}}{C_{3}}, \\
a_{1}(t)=C_{1}, \lambda_{1}(t)=C_{2}, \\
A(t)=\frac{1}{2} \frac{C_{1}^{2} B(t) R}{C_{3}^{2}}, p(t)=C_{3}, \\
\lambda_{0}(t)=\frac{1}{2}\left[C_{1}{ }^{2} R^{2} \int B(t) \mathrm{d} t C_{3}{ }^{2}-C_{1}{ }^{2} R \int B(t) \mathrm{d} t C_{2}{ }^{2}\right. \\
\left.\quad+2 C_{4} C_{3}{ }^{2}\right] / C_{3}{ }^{2},
\end{gathered}
$$

where $R$ and $C_{1}, \cdots, C_{5}$ are all arbitrary constants.

Case 3. In this case, $A(t)$ is considered to be an arbitrary function of $t$.

$$
\begin{aligned}
& a_{0}(t)=\lambda_{2}(t)=0, \lambda_{1}(t)=C_{2}, \quad b_{1}(t)=C_{1}, \\
& q=-2 C_{3} C_{2} \int A(t) \mathrm{d} t+C_{5}, \\
& p(t)=C_{3}, \quad B(t)=-\frac{A(t) C_{3}^{2}}{2 C_{1}^{2}}, \\
& \lambda_{0}=-\frac{1}{2} \int A(t) \mathrm{d} t C_{3}^{2} R-\int A(t) \mathrm{d} t C_{2}^{2}+C_{6}, \\
& a_{1}(t)= \pm \frac{\sqrt{R\left(-1+\mu^{2}\right)} C_{1}}{R},
\end{aligned}
$$

where $R, \mu, C_{1}, C_{3}, C_{5}$ and $C_{6}$ are arbitrary constants.

Case 4. In this case, $\lambda_{2}(t)$ is considered to be an arbitrary function of $t$.

$$
\begin{aligned}
& a_{0}(t)=0, A(t)=-\frac{1}{4} \frac{\lambda_{2 t}}{\lambda_{2}^{2}(t)}, \quad b_{1}(t)=C_{1}, \\
& q(t)=\frac{1}{2} C_{2} C_{3} \lambda_{2}(t)+C_{4}, \lambda_{1}(t)=C_{2} \lambda_{2}(t), \\
& a_{1}(t)= \pm \frac{\sqrt{R\left(-1+\mu^{2}\right)} C_{1}}{R}, B(t)=\frac{1}{8} \frac{C_{3}^{2} \lambda_{2 t}}{C_{1}^{2}}, \\
& \lambda_{0}(t)=\frac{1}{8} \lambda_{2} C_{3}^{2} R+\frac{1}{4} C_{2}^{2} \lambda_{2}(t)+C_{6}, \\
& p(t)=C_{3} \lambda_{2}(t),
\end{aligned}
$$

where $\lambda_{2 t}=\frac{\mathrm{d} \lambda_{2}(t)}{\mathrm{d} t}, C_{1}, C_{2}, C_{3}, C_{4}$ and $C_{6}$ are all arbitrary constants.
Therefore from (5), (6), (8), (11), (12) and (34)(37), we obtain four families of exact solutions for VCNLS equation (7) as follows:

Family 1. From Case 1, the VCNLS equation

$$
i \psi_{t}-\frac{1}{2} \frac{C_{1}^{2} B(t)}{C_{3}^{2}} \psi_{x x}+B(t)|\psi|^{2} \psi=0
$$

where $B(t)$ is an arbitrary function of $t, C_{1}$ and $C_{3} \neq 0$ are arbitrary constants, has the following soliton-like solutions

$$
\begin{gathered}
\psi_{11}(x, t)=C_{1} \sqrt{R} \tanh \left\{\sqrt{R}\left[C_{3} x+q(t)\right]\right\} \\
\cdot \exp \left\{i\left[C_{2} x+\lambda_{0}(t)\right]\right\}, \\
\psi_{12}(x, t)=C_{1} \sqrt{R} \operatorname{coth}\left\{\sqrt{R}\left[C_{3} x+q(t)\right]\right\} \\
\cdot \exp \left\{i\left[C_{2} x+\lambda_{0}(t)\right]\right\},
\end{gathered}
$$

where $C_{2}$ is an arbitrary constant, $q(t)$ and $\lambda_{0}(t)$ are determined by (34).

Family 2. From Case 2, the VCNLS equation

$$
i \psi_{t}+\frac{1}{2} \frac{C_{1}^{2} B(t) R}{C_{3}^{2}} \psi_{x x}+B(t)|\psi|^{2} \psi=0
$$

where $B(t)$ is an arbitrary function of $t, C_{1}$ and $C_{3} \neq 0$ are arbitrary constants, has the following soliton-like solutions

$$
\begin{array}{r}
\psi_{21}(x, t)=C_{1} R \operatorname{sech}\left\{\sqrt{R}\left[C_{3} x+q(t)\right]\right\} \\
\cdot \exp \left\{i\left[C_{2} x+\lambda_{0}(t)\right]\right\}, \\
\psi_{22}(x, t)=C_{1} R \operatorname{csch}\left\{\sqrt{R}\left[C_{3} x+q(t)\right]\right\} \\
\cdot \exp \left\{i\left[C_{2} x+\lambda_{0}(t)\right]\right\},
\end{array}
$$

where $C_{2}$ is an arbitrary constant, $q(t)$ and $\lambda_{0}(t)$ are determined by (35).

Family 3. From Case 3, the VCNLS equation

$$
i \psi_{t}+A(t) \psi_{x x}-\frac{A(t) C_{3}^{2}}{2 C_{1}^{2}}|\psi|^{2} \psi=0,
$$

where $A(t)$ is an arbitrary function of $t, C_{1} \neq 0$ and $C_{3}$ are arbitrary constants, has the following soliton-like solutions 


$$
\begin{aligned}
& \psi_{31}(x, t)=\left[ \pm \sqrt{R\left(-1+\mu^{2}\right)} C_{1} \frac{\operatorname{sech}(\sqrt{R} \xi)}{\mu \operatorname{sech}(\sqrt{R} \xi)+1}+\frac{C_{1} \tanh (\sqrt{R} \xi)}{\mu \operatorname{sech}(\sqrt{R} \xi)+1}\right] \exp \left\{i\left[C_{2} x+\lambda_{0}(t)\right]\right\} \\
& \psi_{32}(x, t)=\left[ \pm \sqrt{R\left(-1+\mu^{2}\right)} C_{1} \frac{\operatorname{csch}(\sqrt{R} \xi)}{\mu \operatorname{csch}(\sqrt{R} \xi)+1}+\frac{C_{1} \operatorname{coth}(\sqrt{R} \xi)}{\mu \operatorname{csch}(\sqrt{R} \xi)+1}\right] \exp \left\{i\left[C_{2} x+\lambda_{0}(t)\right]\right\}
\end{aligned}
$$

where

$$
\xi=C_{3} x-2 C_{3} C_{2} \int A(t) \mathrm{d} t+C_{5}, \quad \lambda_{0}(t)=-\frac{1}{2} \int A(t) \mathrm{d} t C_{3}^{2} R-\int A(t) \mathrm{d} t C_{2}^{2}+C_{6},
$$

$C_{2}, C_{5}$ and $C_{6}$ are arbitrary constants.

Family 4. From Case 4, the VCNLS equation

$$
i \psi_{t}-\frac{1}{4} \frac{\lambda_{2 t}}{\lambda_{2}^{2}(t)} \psi_{x x}+\frac{1}{8} \frac{C_{3}^{2} \lambda_{2 t}}{C_{1}^{2}}|\psi|^{2} \psi=0
$$

where $\lambda_{2}(t) \neq 0$ is an arbitrary function of $t, C_{1} \neq 0$ and $C_{3}$ are arbitrary constants, $\lambda_{2 t}=\mathrm{d} \lambda_{2}(t) / \mathrm{d} t$, has the following soliton-like solutions

$$
\begin{aligned}
& \psi_{41}(x, t)=\left[ \pm \sqrt{R\left(-1+\mu^{2}\right)} C_{1} \frac{\operatorname{sech}(\sqrt{R} \xi)}{\mu \operatorname{sech}(\sqrt{R} \xi)+1}+\frac{C_{1} \tanh (\sqrt{R} \xi)}{\mu \operatorname{sech}(\sqrt{R} \xi)+1}\right] \exp \left\{i\left[x^{2} \lambda_{2}(t)+x \lambda_{1}(t)+\lambda_{0}(t)\right]\right\} \\
& \psi_{42}(x, t)=\left[ \pm \sqrt{R\left(-1+\mu^{2}\right)} C_{1} \frac{\operatorname{csch}(\sqrt{R} \xi)}{\mu \operatorname{csch}(\sqrt{R} \xi)+1}+\frac{C_{1} \operatorname{coth}(\sqrt{R} \xi)}{\mu \operatorname{csch}(\sqrt{R} \xi)+1}\right] \exp \left\{i\left[x^{2} \lambda_{2}(t)+x \lambda_{1}(t)+\lambda_{0}(t)\right]\right\}
\end{aligned}
$$

where

$\xi=C_{3} \lambda_{2}(t) x+\frac{1}{2} C_{2} C_{3} \lambda_{2}(t)+C_{4}$,

$\lambda_{1}(t)=C_{2} \lambda_{2}(t), \quad \lambda_{0}(t)=\frac{1}{8} \lambda_{2} C_{3}^{2} R+\frac{1}{4} C_{2}^{2} \lambda_{2}(t)+C_{6}$,

$C_{2}, C_{5}$ and $C_{6}$ are arbitrary constants.

Now we give a short discussion of our results for Eq. (7). For simplicity, here we only discuss the solution $\psi_{21}$ of (7) under some suitable selections of $A(t)$ and $B(t)$.

1) From $\psi_{21}(x, t)$ in Family 2 , if setting $B(t)=t^{n}$, we can deduce the equation (see [15])

$i \psi_{t}+\frac{1}{2} \frac{C_{1}^{2} R}{C_{3}^{2}} t^{n} \psi_{x x}+t^{n}|\psi|^{2} \psi=0$

has the soliton-like solutions

$$
\begin{gathered}
\psi(x, t)=C_{1} R \operatorname{sech}\left\{\sqrt{R}\left[C_{3} x-\frac{C_{1}^{2} R C_{2}}{(n+1) C_{3}} t^{n+1}+\tilde{C}_{5}\right]\right\} \\
\cdot \exp \left\{i\left[C_{2} x+\frac{C_{1}^{2} R\left(R C_{3}^{2}-C_{2}^{2}\right)}{2 C_{3}^{2}(n+1)} t^{n+1}+\tilde{C}_{4}\right]\right\}, \quad(51)
\end{gathered}
$$

where $R, C_{1}, C_{2}, C_{3}, \tilde{C_{4}}$ and $\tilde{C}_{5}$ are all constants.
2) The conventional stable nonlinear Schrödinger (NLS) equation (see $[1,15]$ ) reads

$$
i \psi_{t}+\psi_{x x}+2|\psi|^{2} \psi=0
$$

which plays an ubiquitous role in physics. It arises as an asymptotic limit of a slowly varying dispersive wave envelope in a nonlinear medium and as such has significant applications; e.g. nonlinear optics, water waves, plasmas physics, etc. From (42), we obtain the following solution of the NLS equation (52):

$$
\begin{aligned}
\psi(x, t)=C_{1} R \operatorname{sech}\left\{C_{3} \sqrt{R}\left[x-4 C_{2} t+\tilde{C}_{5}\right]\right\} \\
\cdot \exp \left\{i\left[C_{2} x+2\left(R^{2} C_{3}^{2}-C_{2}^{2}\right) t+\tilde{C}_{4}\right]\right\},
\end{aligned}
$$

where $C_{1}^{2} / C_{3}^{2}=2, R, C_{1}, C_{2}, C_{3}, \tilde{C}_{4}$ and $\tilde{C}_{5}$ are all constants.

3) As some illustrative samples, plots of $\left|\psi_{11}(x, t)\right|$, $\left|\psi_{21}(x, t)\right|,\left|\psi_{31}(x, t)\right|$ and $\left|\psi_{41}(x, t)\right|$ with various parameters are given in Figs. 1-4, respectively.

The fundamental set of soliton solutions can be expressed by trigonometric and hyperbolic functions. Therefore we assume the variable coefficients 

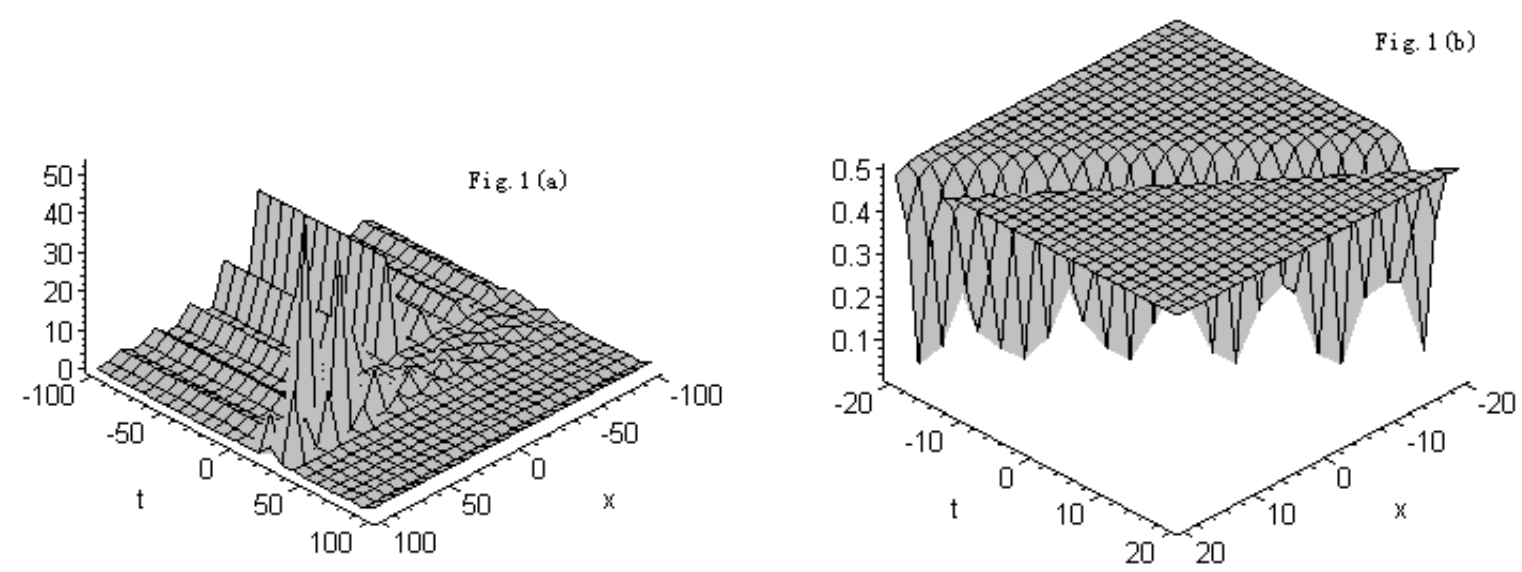

Fig. 1. Plots of $\left|\psi_{11}(x, t)\right|$ with $C_{1}=C_{2}=C_{3}=C_{4}=C_{5}=1 . B(t)=\exp (t), \int B(t) \mathrm{d} t=\exp (t), R=-1 / 4$ in Fig.1(a), $B(t)=1+0.5 \sin ^{2}(t), \int B(t) \mathrm{d} t=\frac{5}{4} t-\frac{1}{8} \sin (2 t), R=\frac{1}{4}$ in Fig. 1(b).
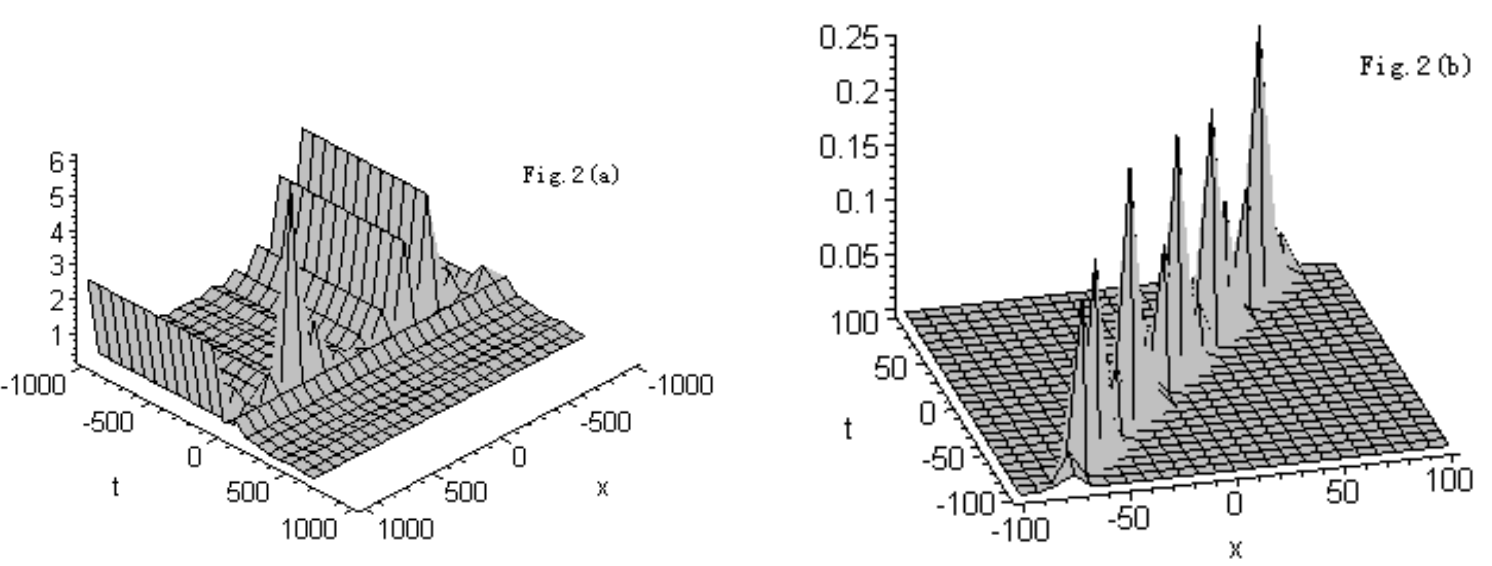

Fig. 2. Plots of $\left|\psi_{21}(x, t)\right|$ with $C_{1}=-1, C_{2}=10, C_{3}=2, C_{4}=-3, C_{5}=1 . B(t)=\exp (t), \int B(t) \mathrm{d} t=\exp (t), R=-1 / 4$ in Fig.2(a), $B(t)=1+0.5 \sin ^{2}(t), \int B(t) \mathrm{d} t=\frac{5}{4} t-\frac{1}{8} \sin (2 t), R=\frac{1}{4}$ in Fig. 2(b).

$A(t), B(t), \lambda_{2}(t)$ (Note: $A(t), B(t)$ in $\psi_{41}(x, t)$ depend on $\left.\lambda_{2}(t)\right)$ of the VCNLS equation (7) is a periodically varying control function: $\alpha+\beta \sin ^{m} k t$. From the figures: 1(b), 2(b), 3(b) and 4(b) we can see that the solutions obtained possess solitonlike features. When $A(t), B(t), \lambda_{2}(t)$ are exponential functions or polynomial functions, from Figs. 1(a), 2(a), 3(a) and 4(a), the solutions $\psi_{11}(x, t), \psi_{21}(x, t)$ do not possess solitonlike features, but the solitonlike features of $\psi_{31}(x, t)$, $\psi_{41}(x, t)$ are not affected greatly.

\section{Summary and Discussion}

In this paper, the improved projective Riccati system method is proposed by introducing coefficients functions' and non-travelling wave' transformation. The proposed method is more powerful than the tanh method, sine-cosine method, generalized hyperbolic function method and generalized Riccati equation expansion method. Making use of the proposed method, we have obtained four families of exact soliton-like solutions of the VCNLS equation with the help of the symbolic computation system-Maple. The results of this paper are of general physical interest, should be readily experimentally verified and might have significant impact on future research. The method proposed here can be applied to other PDEs and related ones.

Acknowledgements: The authors express their sincere thanks to the editors and the referees for their valuable suggestions and kind help. 

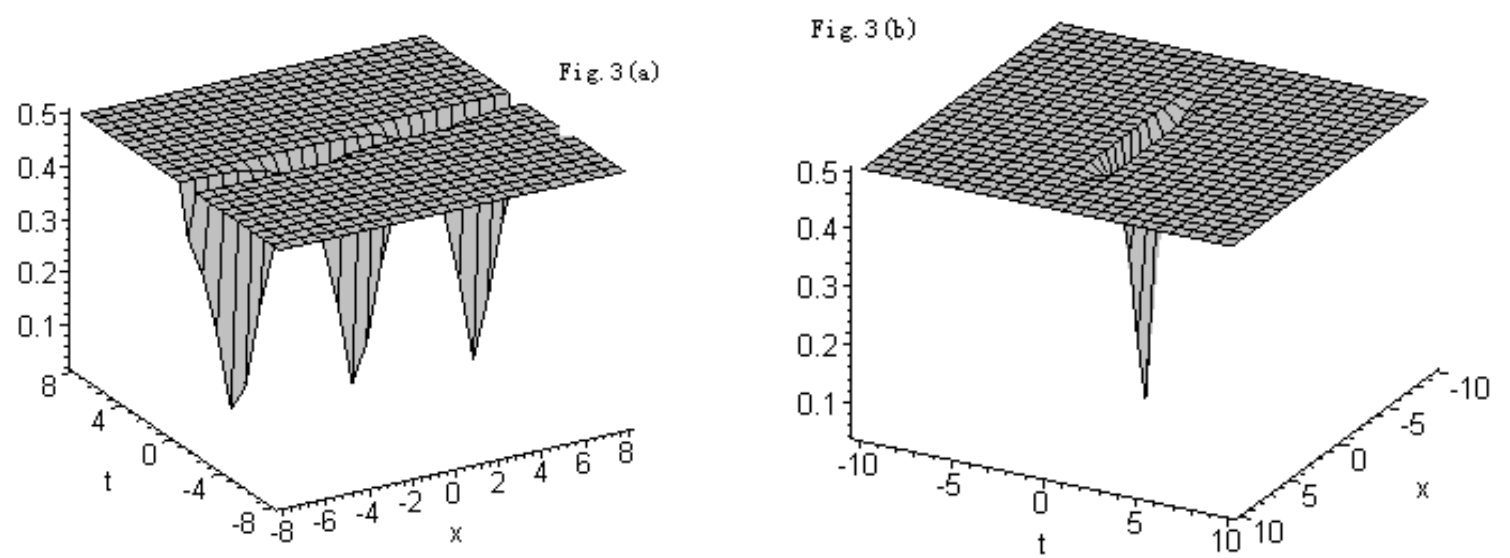

Fig. 3. Plots of $\left|\psi_{31}(x, t)\right|$ with $C_{1}=-1, C_{2}=10, C_{3}=2, C_{4}=-3, C_{5}=C_{6}=1, R=\frac{1}{4}, \mu=2 . A(t)=3 t^{2}, \int A(t) \mathrm{d} t=t^{3}$ in Fig.3(a), $A(t)=1+0.8 \sin ^{2}(t), \int A(t) \mathrm{d} t=1.4 t-0.2 \sin (2 t)$ in Fig. 3(b).
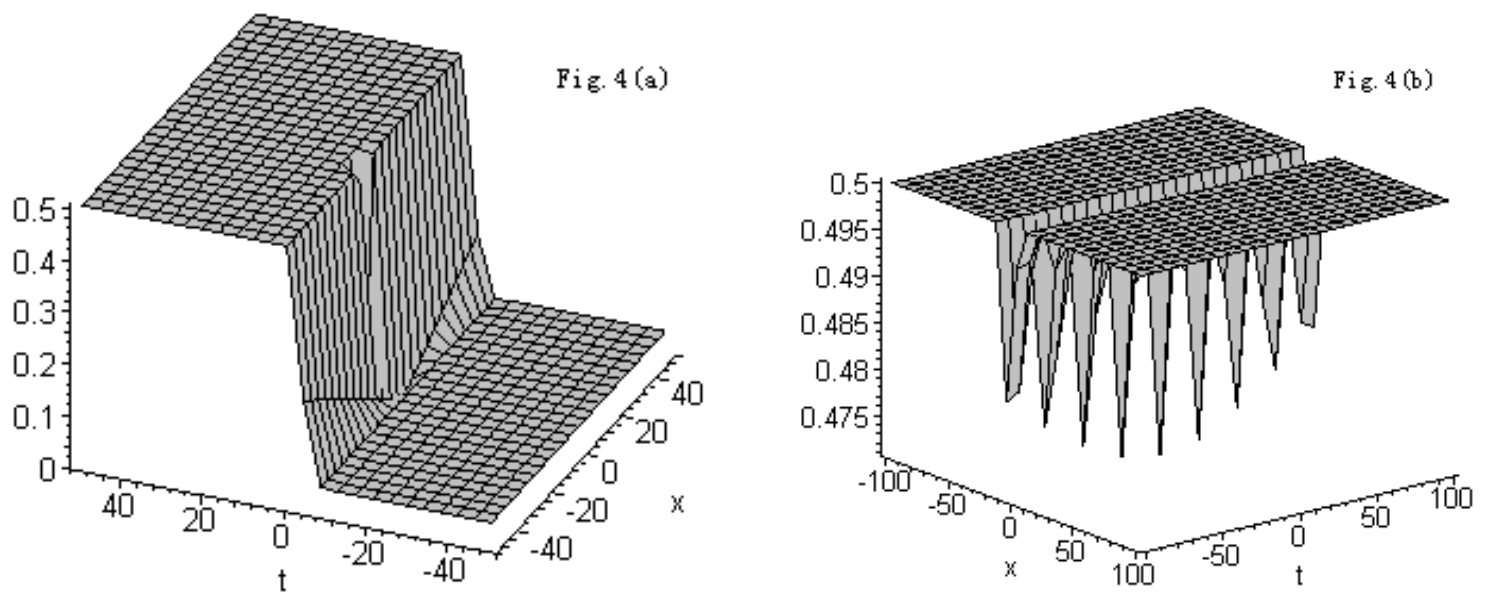

Fig. 4. Plots of $\left|\psi_{41}(x, t)\right|$ with $C_{1}=-1, C_{2}=10, C_{3}=2, C_{4}=-3, C_{5}=C_{6}=1, R=1 / 4, \mu=2 . \lambda_{2}=\exp (t)$ in Fig.4(a), $\lambda_{2}=1+0.5 \sin ^{2}(t)$ in Fig. $4(b)$.

[1] C.H. Gu etc. Soliton theory and its application. Zhejiang Science and Technology Press, Zhejiang 1990.

[2] C. T. Yan, Phys. Lett. A 224, 77 (1996).

[3] Z. Y. Yan and H. Q. Zhang, Appl. Math. Mech. 21, 382 (2000).

[4] E. J. Parkes and B. R. Duffy, Compt. Phys. Commun. 98, 288 (1996).

[5] B. Li, Y. Chen, and H. Q. Zhang, Z. Naturforsch. 57a, 874 (2002).

[6] E. Fan, Phys. Lett. A 277, 212 (2000).

[7] Z. Y. Yan, Phys. Lett. A 292, 100 (2001).

[8] H. N. Xuan and B. Li, Z. Naturforsch. 58a, 167 (2003); B. Li, Y. Chen, H.N. Xuan, and H. Q. Zhang, Chaos, Solitons and Fractals 17, 885 (2003).
[9] Y. T. Gao and B. Tian, Comput. Phys. Commun. 133, 158 (2001); B. Tian and Y. T. Gao, Z. Naturforsch. 57a, 39 (2002).

[10] R. Conte and M. Musette, J. Phys. A 25, 5609 (1992).

[11] T. C. Bountis, V. Papageorgiou, and P. Winternitz, J. Math. Phys. 27, 1215 (1986).

[12] G. X. Zhang, Z. B. Li, and Y. S. Duan, Sci. Chin. Ser. A 44, 396 (2001).

[13] Z. Y. Yan, Chaos, Solitons and Fractals 16, 759 (2003).

[14] B. Li and Y. Chen, Z. Naturforsch. 58a, 511 (2003).

[15] A. H. Khater, M.H. M. Moussa, and S. F. Abdul-Aziz, Chaos, Solitons and Fractals 15, 1 (2003). 